Revue d'histoire de l'Amérique française

ALS REVUE D.HISTOIRE DE L'AMÉRIQUE FRANÇAISE

\title{
Aperçu sur les élections provinciales du Québec de 1867 à 1886
}

\section{Marcel Caya}

Volume 29, numéro 2, septembre 1975

URI : https://id.erudit.org/iderudit/303441ar

DOI : https://doi.org/10.7202/303441ar

Aller au sommaire du numéro

Éditeur(s)

Institut d'histoire de l'Amérique française

ISSN

0035-2357 (imprimé)

1492-1383 (numérique)

Découvrir la revue

Citer cet article

Caya, M. (1975). Aperçu sur les élections provinciales du Québec de 1867 à

1886. Revue d'histoire de l'Amérique française, 29(2), 191-208.

https://doi.org/10.7202/303441ar d'utilisation que vous pouvez consulter en ligne.

https://apropos.erudit.org/fr/usagers/politique-dutilisation/ 


\title{
APERÇU SUR LES ELECTIONS PROVINCIALES DU QUEBBEC DE $1867 \AA 1886$ *
}

\author{
Marcel Caya \\ Archives publiques \\ du Canada, Ottawa
}

Le parti pris presque exclusif manifesté par les historiens politiques canadiens pour des explications se référant à des caractéristiques idéologiques a sérieusement limité le champ d'exploration de l'histoire de la politique canadienne. L'étude du phénomène électoral comme tel a généralement consisté à résumer les débats soutenus par les politiciens les plus représentatifs et à les rapprocher des résultats globaux de l'élection afin d'évaluer quelle question électorale a constitué le facteur déterminant dans la victoire de tel ou tel parti. ${ }^{1}$ En plus de faire de la politique une activité intellectuelle définie où succès et défaites s'expliquent logiquement et tangiblement par les origines, les idées et les talents particuliers des parties en présence, ce schéma général d'interprétation est directement dépendant des sources manuscrites et imprimées utilisées par l'historien et limite son étude aux rôle et perceptions de l'homme politique sans vraiment rendre compte du contexte général dans lequel il a évolué. Utilisée dans le cadre d'une réflexion sur le phénomène électoral, cette approche fait de l'électorat un genre de tribunal jugeant chaque candidat aux arguments présentés sans tenir compte de la composition du jury.

Bien que les débats électoraux ou les enjeux d'une campagne donnée puissent avoir une certaine importance sur l'issue, il est impossible qu'ils aient eu le poids qu'on leur a attribué dans la

\footnotetext{
* Version remaniée d'une communication présentée sous le même titre à la rencontre des Sociétés savantes, à Edmonton, le 7 juin 1975 .

L'auteur désire remercier vivement les professeurs Paul D. Stevens, David Hoffman et André Blais qui ont lu une première version de cet article et fourni de nombreux commentaires utiles.

1 Les exemples les plus souvent utilisés sont J. M. Beck, Pendulum of Power, Canada's Federal Elections (Scarborough, Prentice-Hall, 1968); Jean Hamelin, Jacques Letarte, Marcel Hamelin, "Les élections provinciales dans le Québec", Cahiers' de Géographie de Québec, IV (7 octobre, 1959 - mars 1960) : 5-207; Jean Hamelin, John Huot, Marcel Hamelin, Aperçu de la politique canadienne au XIX siècle (Québec, Culture, 1965); R. Rumilly, Histoire de la Province de Québec (Montréal, Valiquette, 19401943), tomes I à V.
}

RHAF, vol. 29, no 2 (septembre 1975) 
détermination des résultats globaux. Une élection générale est, avant tout, une joute où deux équipes de candidats avec des organisations et des programmes différents, s'affrontent dans un nombre défini de circonscriptions dans le but de faire élire le plus grand nombre possible de candidats afin de pouvoir former le prochain gouvernement. Le succès de l'une ou l'autre équipe partisane dépend, en partie, de l'attrait exercé sur l'électorat par les solutions proposées par tel ou tel parti; il dépend surtout des moyens dont dispose un parti politique pour faire passer son message et amener les électeurs qui l'appuient à enregistrer cette approbation en allant voter.

Parce que l'interprétation des résultats électoraux de la période a été négligée ou, du moins, peu discutée, il a été jusqu'ici difficile de caractériser avec quelque précision le phénomène électoral afin de recréer, au moins dans leurs grandes lignes, les limites réelles des combats politiques de la fin du XIXe siècle. Lorsqu'elles ont dépassé une considération globale des résultats électoraux à l'échelle de la province, les études qui ont abordé la question du phénomène électoral québécois, ont surtout insisté sur l'importance des facteurs régional et religieux. ${ }^{2}$ Pour plus d'un, O. D. Skelton en tête ${ }^{3}$, le sort du parti libéral ne s'améliore qu'à partir du discours de Laurier sur le libéralisme politique en 1877 alors que, pour la plupart, c'est surtout l'utilisation de l'affaire Riel par Honoré Mercier qui aurait fait des libéraux une nouvelle force politique au Québec. ${ }^{4}$ Ces interprétations n'ont pas tenu compte, ni de la victoire libérale au niveau provincial de 1878 avec, à sa tête, le protestant Joly de Lotbinière, ni de l'effritement progressif des forces conservatrices au cours de la période. Il nous semble ici important d'insister sur le caractère plus proprement politique de la lutte électorale au Québec de 1867 à 1886, afin de déterminer jusqu'à quel point l'utilisation des statistiques électorales peut servir à expliquer les résultats des élections générales de la période. Même si, comme l'ont répété nombre de sociologues, le vote n'est toujours qu'un indicateur superficiel de

2 Entre autres, R. W. Cox, The Quebec Provincial Election of 1886, thèse de M. A., (Université McGill, 1948); Philip E. Shea, Electoral Practices in Quebec, 1867-1882, thèse de M. A. (Université McGill, 1968) ; P. B. Waite, Canada, 1874-1896. Arduous Destiny (Toronto, McClelland and Stewart, 1971), 188; Robert Rumilly, Histoire de la Province de Québec (Montréal, Valiquette, 1940-1943), tomes I à V; Jean Hamelin, Jacques Letarte et Marcel Hamelin, op. cit.

3 Oscar Douglas Skelton, Life and Letters of Sir Wilfrid Laurier, edited by David M. L. Farr (Toronto, McClelland and Stewart, 1965), 2 vols.

4 Mason Wade, The French Canadians, 1760-1967. Edition revisée (Toronto, Macmillan, 1968), volume I (1760-1911): 417-422. 
l'opinion, il n'en est pas moins, pour le politicologue et l'historien politique, le seul instrument d'analyse du processus de sélection des représentants dans un système électif; à ce titre il convient de situer le niveau d'analyse au-delà des résultats globaux pour en étudier les composantes.

Les élections provinciales du Québec de 1867 à 1886 offrent un terrain d'analyse unique en histoire canadienne. Le peu de changement apporté aux limites des circonscriptions électorales facilite grandement les comparaisons entre comtés d'une élection à l'autre..$^{5}$ Cette absence de modifications radicales de la carte électorale permet en outre de comparer les comtés entre eux avec d'autant plus de pertinence que, même si l'ordre chronologique est brisé, la dimension historique est respectée. Même si le parti conservateur domine nettement au cours de toute la période, il est intéressant d'observer la montée graduelle des libéraux non pas jusqu'en 1886 comme l'ont perçu la plupart des auteurs, mais surtout jusqu'en 1878 , date de la première victoire libérale provinciale au Québec (Tableau I).

\section{TABLEAU I}

Nombre de députés élus pour chaque parti aux élections générales provinciales du Québec de 1867 à 1886

$\begin{array}{ccc} & \text { Libéraux } & \text { Conservateurs } \\ 1867 & 13 & 51 \\ 1871 & 17 & 58 \\ 1875 & 22 & 43 \\ 1878 & 31^{6} & 34 \\ 1881 & 13 & 52 \\ 1886 & 36^{7} & 29\end{array}$

5 A toutes fins utiles, seules les circonscriptions de Montréal-Ouest et Montréal-Centre ont subi des changements appréciables.

6 Même s'ils s'étaient présentés candidats conservateurs et avaient été élus comme tels, W. E. Price du comté de Chicoutimi \& Saguenay et Arthur Turcotte du comté de Trois-Rivières, ont donné leur appui à Joly de Lotbinière l'aidant ainsi à se maintenir au pouvoir pendant près de deux ans.

7 Est considéré comme libéral, Alexander Cameron, le seul libéral à ne pas avoir signé la pétition demandant que Mercier soit appelé à former le gouvernement, voir R. Rumilly, Histoire de la Province de Québec (Montréal, Valiquette, 1941), tome V: Riel: 206n. Sont également inclus dans le groupe libéral, les conservateurs-nationalistes qui se sont rangés du côté d'Honoré Mercier, à la suite de leur protestation contre le parti conservateur causée, entre autres choses, par l'affaire Riel. 
L'objectif ne consiste pas à chercher une explication unique qui vaudrait pour les résultats électoraux de tous les comtés au cours de la période entière, ni même d'analyser de façon plus détaillée l'une après l'autre les élections au niveau des comtés. Il s'agit, à ce stade-ci, de dégager des résultats électoraux un aperçu sur les élections provinciales de la période qui tienne compte à la fois de la dimension historique et de la conjoncture définie par ces résultats.

La technique de l'analyse hiérarchique, bien que peu employée jusqu'ici dans la recherche historique, constitue un instrument de recherche précieux pour toute analyse électorale préliminaire. Au Canada, le politicologue Vincent Lemieux est celui qui l'a surtout utilisée dans ses recherches sur le phénomène électoral au Québec et qui en a justifié l'utilisation pour l'analyse des résultats électoraux. ${ }^{8}$ Non seulement la technique permet-elle de "vérifier si l'hypothèse qu'un certain domaine, défini en termes généraux, est unidimensionnel", mais encore elle aide à déterminer "si un certain nombre de comportements bien déterminés constituent une échelle" et fournit "un instrument de mesure permettant d'ordonner" des résultats donnés "selon une certaine variable". ${ }^{9}$ En ordonnant les résultats électoraux selon la majorité des sièges obtenus par le parti gagnant à chaque élection générale, elle permet de briser l'ordre chronologique généralement utilisé comme facteur d'explication historique privilégié et de fournir un outil, - moins encombrant que la superposition de cartes géographiques, - pour évaluer le degré de sûreté pour un parti ou l'autre des sièges électoraux durant une période donnée. La méthode en elle-même est relativement simple (Tableau 2). Il s'agit de partager en deux groupes les victoires libérales et conservatrices (exprimées par un " $x$ ", dans les moitiés libérales ou conservatrices de l'échelle, selon le cas), ordonnant les résultats de façon verticale, selon le nombre croissant de pluralités obtenues par le parti libéral et, de façon horizontale, selon les victoires obtenues par chaque parti dans chacun des comtés. Afin d'ajouter une dimension supplémentaire à l'échelle,

8 Vincent Lemieux, "L'analyse hiérarchique des résultats électoraux", Revue canadienne de Science politique, 1, 1 (mars 1968) : 40-54. - "Les élections provinciales dans le comté de Lévis, de 1912 à 1960", Recherches sociographiques, 11, \#3-4 (juillet-décembre 1961): 367-399. Une version légèrement remaniée du premier article cité est parue dans le volume du mểme auteur: Le quotient politique vrai. Le vote provincial et fédéral au Québec (Québec, les Presses de l'Université Laval, 1973), 167-191.

9 Benjamin Matalon, L'Analyse hiérarchique (Paris, Gauthier-Villars, $1965), 28$, cité dans Vincent Lemieux, "L'analyse hiérarchique des résultats électoraux", in idem, Le quotient politique vrai, 168. 
les élections remportées par acclamation ont été indiquées, ce qui permet de situer la fréquence du phénomène par rapport au degré de sûreté pour un parti ou l'autre des comtés où il se produit. Ce degré de sûreté est défini par la position que le comté occupe dans l'échelle qui, à cette fin, a été divisée en 7 groupes de comtés selon la fréquence des victoires, réelles ou attendues, d'un parti ou l'autre.10 L'échelle parfaite serait celle où les comtés se détachent d'un parti ou l'autre par groupes à l'intérieur d'un couloir défini. Devient erreur, tout résultat qui s'inscrit à l'extérieur de ce couloir limité par l'ensemble des résultats puisqu'il apparaît alors différent du modèle général défini par l'ensemble des résultats.

A l'exemple de celle de Vincent Lemieux, l'échelle des circonscriptions provinciales au Québec de 1867 à 1886 a été modifiée autant que possible de façon à minimiser le nombre d'erreurs en situant les comtés pour lesquels il était nécessaire de faire un choix à un rang supérieur, lorsque les victoires libérales étaient plus nombreuses, et à un rang inférieur, lorsqu'elles l'étaient moins, de façon à distinguer les comtés de tendance libérale de ceux de tendance conservatrice; autant que possible les acclamations ont été situées à l'intérieur du couloir. C'est ce qui explique que des comtés comme ceux de Huntingdon et de Montréal-Ouest se retrouvent à un rang aussi élevé dans l'échelle et que d'autres comme celui d'Argenteuil et de Témiscouata aient été placés si bas. Le rang particulier d'un comté à l'intérieur de l'échelle est, ici, beaucoup moins important que les rapprochements que l'échelle permet d'établir entre comtés dont les résultats globaux ont été similaires.

Bien que le fait que cette étude ne soit basée que sur les résultats de six élections générales (on reconnaît généralement qu'un minimum de dix groupes d'observations est requis) ne permette pas d'en arriver scientifiquement à des conclusions de même type que celles de Vincent Lemieux, il est quand même intéressant de constater que l'échelle produite ne comprend que 35 erreurs sur un total de 389 observations produisant un coeffi-

10 Dans ses analyses hiérarchiques, le professeur Lemieux utilise le rang des comtés dans l'échelle comme outil d'analyse. Parce que, pour notre objet, le nombre de comtés sûrs était proportionnellement élevé et pour insister sur la corrélation de la position d'un groupe de comtés dans l'échelle avec le nombre d'acclamations, nous avons préféré insister moins sur le rang particulier d'un comté ou l'autre et nous limiter à cette définition de degré de sûreté des comtés pour un parti ou l'autre. 
TABLEAU II

Echelle des circonscriptions provinciales au Québec de 1867-1886**

Victoires

libérales

Victoires

conservatrices

1. Châteauguay

2. Iberville

3. St-Hyacinthe

4. St-Jean

5. Lotbinière

6. Huntingdon

7. Montmagny

8. Verchères

9. Drumm.-Arthab.

10. Montréal-Ouest

11. Montréal-Centre

12. Berthier

13. Napierville

14. Rouville

15. Chambly

16. Shefford

17. Beauce

18. Yamaska

19. Québec-Est

20. Québec-Centre

21. Mégantic

22. Lévis

23. Rimouski

24. Bellechasse

25. Kamouraska

26. Québec-Ouest

27. Portneuf

28. L'Islet

29. Bagot

30. Beauharnois

31. Soulanges

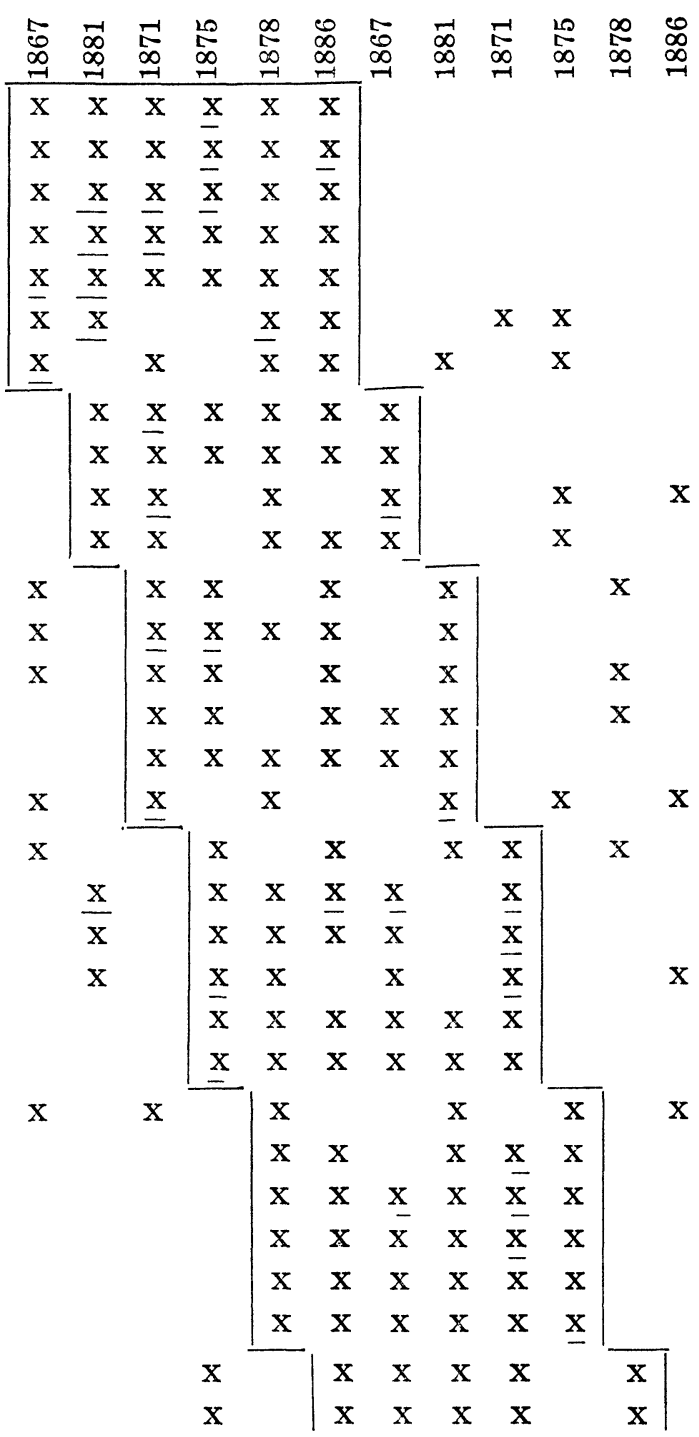


32. Dorchester

33. Champlain

34. Jacques-Cartier

35. L'Assomption

36. Joliette

37. Montréal-Est

38. Richelieu

39. Trois-Rivières

40. Charlevoix

41. Argenteuil

42. Gaspé

43. Stanstead

44. Québec (comté)

45. Montmorency

46. Missisquoi

47. Témiscouata TABLEAU II (suite)
Victoires
conservatrices

Victoires

libérales

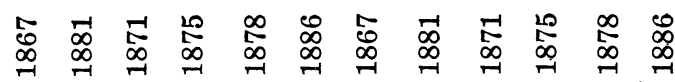

48. Bonaventure

49. Brome

50. Chic.-Saguenay

51. Compton

52. Deux-Montagnes

53. Hochelaga

54. Laprairie

55. Laval

56. Maskinongé

57. Montcalm

58. Nicolet

59. Ottawa

60. Pontiac

61. Richmond \& Wolfe

62. St-Maurice

63. Sherbrooke

64. Terrebonne

65. Vaudreuil

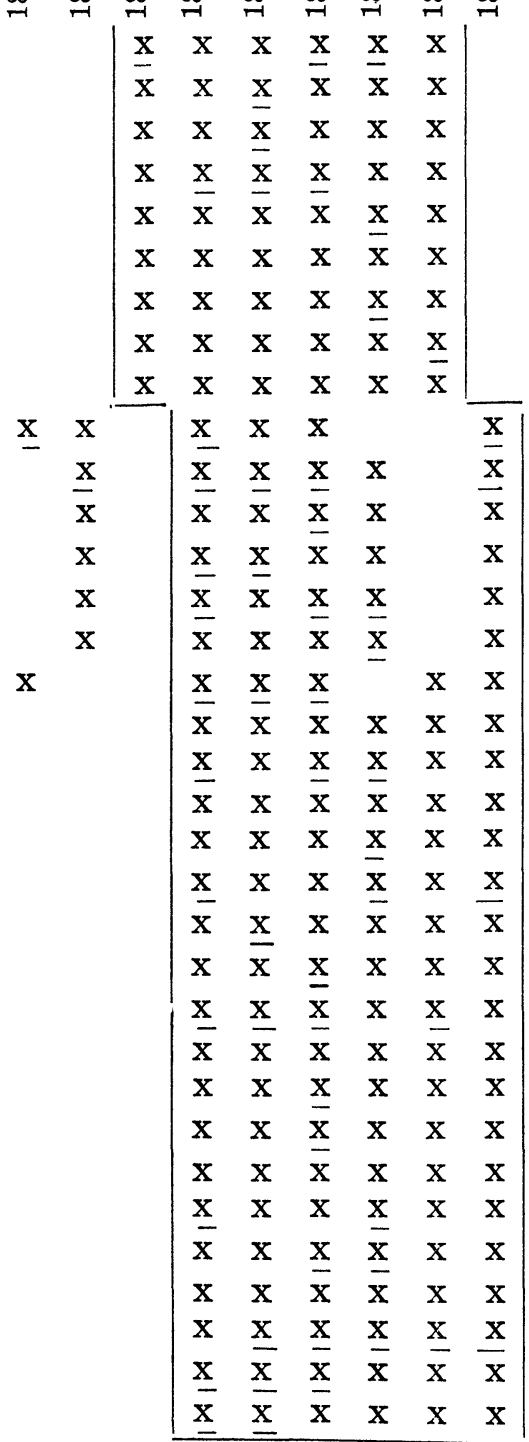

** (Un $\mathrm{x}$ souligné indique une élection par acclamation.) 
cient de reproductibilité de .089 qui est amplement en deçà du pourcentage d'erreurs acceptable de $10 \% .{ }^{11}$

Alors que la plupart des analyses électorales détaillées utilisent comme unité de comparaison soit la région, soit l'origine nationale prédominante de certains comtés, ${ }^{12}$ l'analyse hiérarchique permet de fonder des comparaisons sur des similitudes véritables de comportements des comtés. Ainsi, à l'exception de Montréal-Est et de Trois-Rivières, le parti conservateur a généralement connu des difficultés dans les comtés urbains alors que les comtés semi-urbains d'Hochelaga et de Sherbrooke se sont rangés constamment de son côté. Doit-on y voir une tendance générale des anglophones à voter pour les candidats libéraux? L'hypothèse, bien qu'intéressante, peut difficilement se confirmer quand un aussi grand nombre de circonscriptions rurales où vivent plus de $50 \%$ d'anglophones accordent généralement leurs suffrages aux conservateurs (Brome, Compton, Missisquoi, Pontiac et Stanstead). Il faudra, sans contredit, dépasser ce niveau d'analyse superficiel et tenter de puiser à même une comparaison de caractéristiques socio-économiques et de résultats de paroisses et de cantons, des éléments qui permettraient d'en arriver à des conclusions plus définies.

Il est évident que le niveau d'analyse atteint au moyen de l'analyse hiérarchique ne peut fournir ici que des indications qui, tout intéressantes qu'elles puissent être, n'apportent pas de réponses définitives. Cette technique contribue cependant à orienter toute recherche ultérieure en fournissant certains points de comparaisons fondés sur le comportement de certains comtés, non plus seulement sur leur contexte régional ou leurs caractéristiques religieuse ou ethnique. On peut se demander ainsi pourquoi les comtés de Gaspé, de Stanstead, de Québec, de Montmorency et de Missisquoi ont le même comportement électoral, élisant un libéral en 1878 et un conservateur en 1886. Puisque tous ces comtés, à l'exception de celui de Montmorency, renferment une proportion de population d'origine britannique d'au moins $25 \%$, s'agit-il d'une confirmation de la thèse traditionnelle

11 V. Lemieux, "L'Analyse hiérarchique", 178.

12 Comme entre autres, Marcel Hamelin, Les premières années du parlementarisme québécois (1867-1878) (Québec, Les Presses de l'Université Laval, 1974) ; Jean-Paul Bernard, Les Rouges. Libéralisme, nationalisme et anti-cléricalisme au milieu du XIXe siècle (Montréal, Les Presses de l'Université du Québec, 1971); Philip E. Shea, Electoral Practices in Québec, 1867-1882, thèse de M. A. (McGill University, 1968); R. Cox, The Quebec Provincial Election of 1886. Thèse de M. A. (McGill University, 1948). 
qui a fait de l'utilisation de l'affaire Riel dans la campagne de 1886 de Mercier un obstacle à l'adhésion des anglophones au parti libéral? Doit-on voir une manifestation du même phénomène dans le comportement des comtés francophones de Champlain, Jacques-Cartier, L'Assomption, Joliette, Montréal-Est, Richelieu, Trois-Rivières et Charlevoix qui, pour la première fois en 1886, élisaient des libéraux? On peut quand même se demander pourquoi des comtés comme Montréal-Centre, Shefford et Québec-Ouest, qui renfermaient aussi des proportions importantes d'anglophones, ont maintenu leur allégeance libérale alors que nombre d'autres comtés, entièrement francophones, n'ont pas semblé être affectés par la présumée vague nationaliste qui aurait déferlé sur le Québec au cours de cette campagne de 1886. Il devient aussi évident qu'on ne peut fonder aucune interprétation (ou réinterprétation) globale des résultats électoraux sur les caractéristiques socio-économiques ou régionales des comtés. Si l'on peut en général identifier, à partir de l'échelle, certains noyaux partisans comme, par exemple, les comtés de Châteauguay, Iberville, St-Jean et St-Hyacinthe, qui, de fait, possèdent certaines caractéristiques socio-économiques communes, il demeure quand même difficile d'imputer des schèmes d'explications communs à leur comportement puisque des comtés voisins, comme ceux de Laprairie, Napierville, Beauharnois, partageant les mêmes caractéristiques générales, ont produit des résultats opposés dans la plupart des cas. Seul un fractionnement de l'unité d'analyse permettra de situer le débat sur des bases réalistes.

L'analyse hiérarchique nous permet de mettre à jour certains faits nouveaux et d'identifier certains problèmes qui ont jusqu'ici peu retenu l'attention. En divisant le Tableau II en ses différents échelons et en comparant pour chacun d'eux le nombre d'erreurs et le nombre d'acclamations qu'ils comprennent, on $\mathrm{y}$ remarque une certaine correspondance entre les blocs des élections contestées (des numéros 6 à 47), où le nombre d'erreurs augmente et le nombre d'acclamations est relativement minime, et les blocs des élections sûres, dans les premiers et derniers échelons, où le phénomène contraire se produit.

Alors que les conservateurs ont remporté la victoire en au moins une occasion dans 60 des 65 comtés, les libéraux, pour leur part, ont été impuissants dans 17 comtés, soit dans plus d'un quart du nombre total de circonscriptions. Si on accepte la définition de comtés sûrs conservateurs et libéraux fournie par l'échelle, il n'existerait vraiment que cinq comtés libéraux sûrs où le parti adverse n'a jamais pu l'emporter et dans lesquels il a préféré 
TABLEAU III

Comparaison des proportions d'acclamations et d'erreurs des diverses parties de l'échelle des circonscriptions électorales du Québec 1867 à 18.86

\begin{tabular}{lrrrrrrr} 
Libéraux: & 6 & 5 & 4 & 3 & 2 & 1 & 0 \\
Conservateurs: & 0 & 1 & 2 & 3 & 4 & 5 & 6 \\
Acclamations: & $13 / 42$ & $4 / 24$ & $4 / 36$ & $8 / 35$ & $5 / 35$ & $11 / 66$ & $47 / 150$ \\
& $(.31)$ & $(.17)$ & $(.11)$ & $(.23)$ & $(.14)$ & $(.17)$ & $(.31)$ \\
Erreurs: & $4 / 42$ & $3 / 24$ & $9 / 36$ & $6 / 36$ & $3 / 35$ & $2 / 66$ & $8 / 150$ \\
& $(.10)$ & $(.12)$ & $(.25)$ & $(.17)$ & $(.09)$ & $(.03)$ & $(.05)$ \\
\hline
\end{tabular}

concéder la victoire pratiquement une fois sur trois; les libéraux, pour un bloc correspondant de comtés traditionnellement conservateurs, y ont concédé presque la même proportion d'élections par acclamation (Tableau III).

Il devient évident que les élections générales au Québec n'ont pas toujours été une lutte égale entre deux partis où les corps électoraux de tous les comtés ont eu chance égale de choisir entre les deux principales formations politiques, les conservateurs et les libéraux. Au niveau parlementaire, il a toujours existé un certains bipartisme institutionnel imposé par les cadres du régime parlementaire britannique: au contraire de ce qui s'était produit au cours de la période antérieure, les parlements d'après 1867 sont stables et, malgré la présence de quelques députés qui se sont déclarés indépendants, la polarisation entre parti gouvernemental et parti de l'opposition s'est réalisée très vite. ${ }^{13} \mathrm{Ce}$ bipartisme ne s'est transposé à l'extérieur du cadre parlementaire que très lentement. L'échelle fait ressortir clairement que 22 comtés sur 65 , plus d'un tiers, sont des comtés sûrs pour un parti ou l'autre: 17 pour les conservateurs et 5 pour les libéraux. En plus d'être les comtés où le plus grand nombre de candidats ont été élus par acclamation, ils sont aussi ceux où, généralement, en d'autres occasions, deux candidats d'un même parti ont contesté la même élection. C'est donc dire que, à cause de l'incapacité des libéraux à présenter un nombre suffisant de candidats, l'électorat de chacun des comtés n'a pas eu véritablement de chances égales d'élire le candidat du parti de son choix. A cause de la faiblesse d'organisation des libéraux, le combat politique réel a toujours été limité à une minorité de comtés où les deux partis escomptaient remporter la victoire. Ces comtés sont ceux

13 Marcel Hamelin, dans son étude sur les premières années du parlementarisme québécois, ne reconnaît l'élection d'aucun indépendant en 1867, d'un seul (Andrew Esinhart pour Laprairie) en 1871, d'aucun en 1875. 
où, dans l'échelle, le nombre d'acclamations est le moindre et où, en même temps, les traditions partisanes sont le mieux équilibrées. La domination du parti conservateur au cours de cette période s'explique en grande partie par la faiblesse de l'organisation électorale des libéraux.

C'est pourquoi le problème historique posé par l'étude des élections provinciales du Québec de 1867 à 1886 n'est pas surtout celui de la victoire libérale de 1886. Considérée isolément, elle peut toujours être expliquée par la juxtaposition d'un certain nombre de facteurs déjà abordés dans l'historiographie. On ne peut cependant l'expliquer vraiment sans rendre compte de la victoire de ce même parti en 1878 et de sa faiblesse chronique durant le reste de la période. En dernière analyse, le parti libéral s'est toujours battu lui-même par son incapacité à s'organiser lorsqu'il ne put réussir à présenter de candidats dans un nombre suffisant de circonscriptions. Chaque fois qu'il a concédé un avantage de plus de cinq élections par acclamation à l'adversaire, il a subi d'importantes défaites. Les deux seules fois où il a pu accéder de justesse au pouvoir à l'issue d'une élection, sont celles où il avait su limiter le nombre de ses "cadeaux" au parti adverse. Le tableau IV, en comparant le nombre des acclamations concédées par chaque parti et celui des majorités obtenues par les conservateurs à chaque élection, expose, de façon manifeste, ce handicap que les libéraux durent assumer au début de quatre des six élections de la campagne.

TABLEAU IV

Acclamations concédées par chaque parti aux élections générales provinciales de 1867 à 1886

$$
\begin{gathered}
\text { Candidats élus } \\
\text { par acclamation }
\end{gathered}
$$

1867

1871

1875

1878

1881

1886
$\mathrm{C}$

17

20

12

3

12

4
Majorités conservatrices

\section{DIFFERENCE}

15

38

13

29

21

1

1

39 
Pourtant, en termes de stratégie électorale, il était logique qu'un parti maximise son effort là où il croyait obtenir les meilleurs résultats. Il est logique qu'un parti ne présente des candidats que là où il a quelque chance de remporter la victoire afin d'éviter de dépenser inutilement des ressources souvent maigres en personnel et en argent. Les expériences de 1878 et de 1886 ont démontré, au contraire, une des caractéristiques typiques de l'époque des élections par acclamation: il fallait présenter au moins, le plus grand nombre de candidats possible quitte à réduire l'effort réel au cours de la campagne, - afin d'occuper le plus grand nombre possible de candidats et d'organisations de candidats adverses. L'absence de candidats, en assurant l'élection par acclamation à trop d'adversaires, les laissait libres, en même temps que les membres de leur organisation, d'aller prêter main-forte à d'autres candidats de leur parti en moins bonne posture. Ainsi, la faiblesse d'organisation des libéraux au niveau de certains comtés, non seulement provoquait automatiquement leur défaite dans ces comtés, mais diminuait aussi leurs chances dans les autres circonscriptions à travers la province.

Les élections de 1878 et de 1886, en même temps que des victoires libérales, ont surtout été les élections où les résultats ont été les plus serrés et où la participation des électeurs a atteint ses plus hauts niveaux. Afin de déterminer jusqu'à quel point les résultats d'une élection générale avaient été plus serrés que ceux d'une autre et parce que le nombre d'élections par acclamation était trop grand pour permettre l'utilisation de simples moyennes, les pluralités obtenues par le candidat élu au cours d'élections générales ont dû être comparées d'abord au niveau de chaque comté. En assignant alors à l'intérieur d'un même comté un nombre de points variant de 6 à 1 à chacune des années d'élection générale selon le rang de pluralité de chacune des élections et en additionnant, pour chacune des élections, le produit du nombre de comtés par le score alloué, il est possible de déterminer un rang propre pour chacune des élections et ainsi comparer les pluralités d'une élection générale à l'autre. 


\section{TABLEAU V}

Répartition des pourcentages de pluralités électorales obtenues par les candidats élus aux élections générales provinciales du Québec de 1867 à 1886 selon leur rang

\begin{tabular}{llclcccc} 
& & 1867 & 1871 & 1875 & 1878 & 1881 & 1886 \\
$1^{\circ} \mathrm{rang}$ & $(\mathrm{x} 6)$ & $8(48)$ & $2(12)$ & $6(36)$ & $15(90)$ & $12(72)$ & $22(132)$ \\
$2^{\circ} \mathrm{rang}$ & $(\mathrm{x} 5)$ & $7(35)$ & $6(30)$ & $10(50)$ & $12(60)$ & $12(60)$ & $17(85)$ \\
$3^{\circ} \mathrm{rang}$ & $(\mathrm{x} 4)$ & $4(12)$ & $7(28)$ & $12(48)$ & $16(64)$ & $10(40)$ & $12(48)$ \\
$4^{\circ} \mathrm{rang}$ & $(\mathrm{x} 3)$ & $13(39)$ & $6(18)$ & $9(27)$ & $13(39)$ & $6(12)$ & $4(12)$ \\
$5^{\circ} \mathrm{rang}$ & $(\mathrm{x} 2)$ & $10(20)$ & $9(18)$ & $8(16)$ & $3(6)$ & $5(10)$ & $2(4)$ \\
$6^{\circ} \mathrm{rang}$ & $(\mathrm{x} 1)$ & $3(3)$ & $8(8)$ & $1(1)$ & $1(1)$ & $3(3)$ & $1(1)$ \\
\hline
\end{tabular}

Pour vingt-deux comtés sur soixante-cinq, les résultats sont les plus serrés en 1886 alors que pour quinze comtés en 1878 et pour douze en 1881 le candidat élu a dû partager une plus grande proportion du vote populaire. La compilation globale des résultats du Tableau VI accorde d'emblée à l'élection de 1886, le rang d'élection la plus contestée.

\section{TABLEAU VI}

Rang et score obtenus par chaque élection générale provinciale du Québec de 1867 à 1886 selon leur répartition des pourcentages des pluralités des candidats élus

$\begin{array}{ccc}\text { rang } & \text { élection } & \text { score } \\ 1 & 1886 & 282 \\ 2 & 1878 & 260 \\ 3 & 1881 & 197 \\ 4 & 1875 & 178 \\ 5 & 1867 & 157 \\ 6 & 1871 & 114\end{array}$

Il en est de même pour la participation électorale. Traditionnellement, on obtient les taux de participation électorale en additionnant tous les pourcentages de participation et en divisant le 
total par le nombre de comtés où il y a eu élection. ${ }^{14}$ Parce que ces résultats ne tiennent pas compte des différences des difficultés à enregistrer le vote de chaque comté et qu'ils ne comparent pas les mêmes comtés d'une élection à l'autre, il fallait vérifier ces statistiques d'une manière qui tienne compte de la globalité des résultats en même temps qu'elle comparerait les différents pourcentages de participation d'un même comté. Afin d'y parvenir, la méthode utilisée pour la comparaison des pluralités a été retenue (Voir Tableau VII).

\section{TABLEAU VII}

Répartition des pourcentages de participations électorales aux élections générales provinciales du Québec de 1867 à 1886 selon leur rang

\begin{tabular}{llclclll} 
& & 1867 & 1871 & 1875 & 1878 & 1881 & 1886 \\
$1^{\circ} \mathrm{rang}$ & $(\mathrm{x} 6)$ & $16(96)$ & $6(36)$ & $3(18)$ & $24(144)$ & \multicolumn{1}{l}{0} & $16(96)$ \\
$2^{\circ} \mathrm{rang}$ & $(\mathrm{x} 5)$ & $8(40)$ & $7(35)$ & $7(35)$ & $17(85)$ & $5(25)$ & $22(110)$ \\
$3^{\circ} \mathrm{rang}$ & $(\mathrm{x})$ & $9(36)$ & $4(16)$ & $13(52)$ & $10(40)$ & $12(48)$ & $10(40)$ \\
$4^{\circ} \mathrm{rang}$ & $(\times 3)$ & $6(18)$ & $7(21)$ & $14(42)$ & $5(15)$ & $15(45)$ & $5(15)$ \\
$5^{\circ} \mathrm{rang}$ & $(\mathrm{x})$ & $2(4)$ & $7(14)$ & $8(16)$ & $2(4)$ & $13(26)$ & $4(16)$ \\
$6^{\circ} \mathrm{rang}$ & $(\mathrm{x} 1)$ & $4(4)$ & $6(6)$ & $1(1)$ & $2(2)$ & $3(3)$ & $1(1)$
\end{tabular}

Ainsi, dans vingt-quatre comtés sur soixante-cinq, c'est l'élection de 1878 qui a généré la plus importante participation électorale alors que, à celles de 1867 et de 1886, seulement seize comtés ont atteint respectivement leur plus haut niveau de participation. Par contre, la participation à l'élection de 1886 se

$\begin{array}{cccc}14 & \text { Moyennes de participations électorales aux élections } \\ \text { générales provinciales de } 1867 \text { à } 1886 \\ \text { Moyenne de } \\ \text { Election } & & \text { participation } & \text { Rang } \\ 1867 & \text { N. d'élections } & 69.3 & 3 \\ 1871 & 45 & 60.4 & 6 \\ 1875 & 38 & 64.9 & 4 \\ 1878 & 46 & 69.9 & 1 \\ 1881 & 60 & 62.3 & 5 \\ 1886 & 48 & 69.8 & 2\end{array}$

Jean Hamelin, Jacques Letarte et Marcel Hamelin, op. cit., ont obtenu des résultats similaires, mais, opérant à partir de concepts différents, discutent plutôt d'abstentions. Leur préoccupation est tout autre puisqu'elle s'attache surtout à décrire le long terme en faisant la distinction entre diverses sous-périodes dépassant largement les vingt années couvertes par le présent aperçu. 
situe au deuxième rang pour vingt-deux circonscriptions alors que celle de 17 comtés atteint le même rang en 1878. L'influence sur les résultats globaux de la chute de la participation électorale en 1881 n'en devient que plus évidente et témoigne à coup sûr de l'importance de la dimension plus proprement politique dans la détermination des résultats électoraux de la période.

\section{TABLEAU VIII}

Rang et score obtenus par chaque élection générale provinciale du Québec de 1867 à 1886 selon leur répartition des pourcentages de participation électorale

$\begin{array}{ccc}\text { rang } & \text { élection } & \text { score } \\ 1 & 1878 & 290 \\ 2 & 1886 & 270 \\ 3 & 1867 & 198 \\ 4 & 1875 & 164 \\ 5 & 1881 & 147 \\ 6 & 1871 & 128\end{array}$

De fait, il existe pour toute la période, au niveau des comtés mêmes, une certaine corrélation entre les phénomènes de changement d'allégeance partisane d'un comté et de variation de la participation électorale. Sur un total de 324 changements possibles d'allégeance partisane d'un comté d'une élection générale à une autre, seulement 91 élections (28\% des cas) ont vu l'emporter un candidat d'allégeance différente de celle du vainqueur de l'élection précédente. Pour seulement 18 de ces 91 cas, la variation de la participation électorale d'avec le scrutin précédent était inférieure à $5 \%$; en 20 occasions, cette variation se situait entre 5 et $10 \%$ et 28 fois, elle a dépassé $10 \%$; les 19 autres cas sont ceux où une élection par acclamation a précédé ou suivi un changement d'allégeance (Tableau IX).

Dans un système politique où les variations sont aussi limitées, les traditions partisanes jouent un rôle certain: un comté ne change pas d'allégeance facilement; que 48 de ces changements d'allégeance partisane $(52.7 \%$ du total) aient été produits parallèlement à des variations de participation électorale d'au moins $5 \%$ indique que, sans que les votants habituels des comtés affectés n'aient à voter différemment, la seule addition des votes de ceux qui avaient décidé de s'abstenir à l'élection précédente 
TABLEAU IX

Répartition des changements d'allégeance de comtés selon les variations du taux de participation

\begin{tabular}{lcccc} 
& victoire & \multicolumn{4}{c}{$\begin{array}{c}\text { victoire } \\
\text { par même } \\
\text { candidat } \\
\text { malgré } \\
\text { Variation du } \\
\text { taux de } \\
\text { participation } \\
\text { de parti } \\
\text { différent }\end{array}$} & $\begin{array}{c}\text { vartoire } \\
\text { parti }\end{array}$ & $\begin{array}{c}\text { changement } \\
\text { d'allégeance }\end{array}$ & TOTAUX \\
$\begin{array}{l}\text { p-10\% } \\
\text { plus de 10\% }\end{array}$ & 18 & 51 & 0 & 69 \\
$\begin{array}{l}\text { acclamation } \\
\text { précède ou suit }\end{array}$ & 20 & 33 & 0 & 53 \\
TOTAUX & 19 & 39 & 0 & 67 \\
\hline
\end{tabular}

ou la soustraction des suffrages de ceux qui auraient décidé de s'abstenir peut expliquer le changement d'allégeance partisane d'un comté d'une élection à une autre. C'est pourquoi il est important, dans toute tentative d'explication de résultats électoraux, de considérer que l'activité la plus efficace des politiciens au cours d'une campagne électorale ne se situe pas surtout sur le plan idéologique à tenter de convaincre, par des discours ou des éditoriaux de journaux, les sympathisants du parti adverse, mais bien à amener le plus grand nombre de ses propres sympathisants à se rendre voter. Avant notre ère de la diffusion électronique, seule une organisation électorale solide pouvait mener cette tâche à bien.

Bien que, dans le cadre d'études en histoire sociale et intellectuelle, on doive restreindre les résultats électoraux à un rôle d'indicateurs sans leur assigner de valeur absolue, il semble que l'étude de l'histoire politique de la période doive y puiser ses propres schèmes d'explications. Le fait que, à l'exception de l'élection de 1881, l'ordre chronologique soit celui qui produise le moins d'erreurs dans l'échelle (Tableau II) dénote très bien l'évolution politique graduelle à partir d'une domination entière des conservateurs sur la politique aux lendemains de la Confédération à une situation où le parti libéral est en position de contester et même de renverser cette prédominance. Les gains du parti le plus faible en 1867 ont été progressifs: l'échelle indique clairement qu'à chaque élection, les gains libéraux ont été rarement éphémères et n'ont été accompagnés que de pertes minimales de 
comtés qu'ils possédaient déjà. A l'occasion de leurs plus importants succès, en 1878 et en 1886, les libéraux ont réussi, tout en défendant avec succès la plupart des comtés déjà acquis, à arracher un certain nombre de comtés à l'adversaire. En 1878, ils ont acquis dix nouveaux comtés (Kamouraska, Québec-Ouest, Portneuf, L'Islet, Bagot, Gaspé, Stanstead, Québec, Montmorency et Missisquoi), en n'en perdant temporairement que quatre (Berthier, Rouville, Chambly et Yamaska). En 1886, même s'ils en ont perdu cinq (Gaspé, Stanstead, Québec, Montmorency et Missisquoi) qu'ils avaient acquis temporairement en 1878 en plus de Montréal-Ouest et Mégantic qui leur étaient traditionnellement acquis, ils en ont gagné neuf autres (Dorchester, Champlain, Jacques-Cartier, L'Assomption, Joliette, Montréal-Est, Richelieu, Trois-Rivières et Charlevoix) qui avaient jusque-là maintenu leur allégeance conservatrice, même en 1878. Leur défaite de 1881, accompagnée d'un nombre important d'élections par acclamation et d'une baisse générale de la participation électorale dans les comtés où il y a eu élection, a été provoquée, de toute évidence, par une défaillance complète de l'organisation du parti qui, non seulement n'a pu présenter assez de candidats, mais encore n'a même pas été capable de générer, dans les comtés où il y avait lutte, une participation électorale comparable à celle qui, en 1878 et 1886, avait accompagné leur succès.

L'histoire des élections au Québec de 1867 à 1886 en est une de domination de la scène politique par le parti conservateur; des six élections, ils en ont remporté quatre, ne perdant les deux autres que de justesse (Tableau I). Néanmoins, au milieu de cette domination, se situe un mouvement progressif de contestation politique beaucoup plus important qui se bâtit autour du parti libéral. Bien que le caractère préliminaire des recherches entreprises n'ait pas permis de localiser les traits dominants de cette contestation, il est possible d'affirmer qu'elle n'est pas de nature régionale, ni linguistique. Par contre, les traits dominants de l'évolution de cette contestation sont assez facilement reconnaissables à partir de l'analyse hiérarchique des résultats (Tableau II) : des comtés graduellement arrachés par les libéraux aux conservateurs leur sont restés fidèles en autant que l'organisation électorale libérale a pu s'assurer qu'un nombre maximum de ses sympathisants enregistre leur vote. C'est cette organisation électorale qui, en 1881, avait flanché, incapable de recruter assez de candidats (Tableau IV), ou de générer une participation électorale suffisante (Tableau VII) qui aurait, certes, fait toute la différence dans ces comtés, d'acquisition récente pour les 
libéraux, où la marge du candidat élu fut très réduite (Tableaux $\mathrm{V}$ et VI). En 1886, la situation était revenue à la normale et les libéraux, à l'occasion d'une campagne des plus dures, ont été capables de reprendre le terrain perdu; les conservateurs, de leur côté, ont dû se contenter presqu'exclusivement des comtés qui leur avaient été traditionnellement acquis. Ainsi la victoire électorale de Mercier en 1886 qui, selon l'historiographie, a marqué les débuts des succès des libéraux au Québec, ne peut être expliquée exclusivement en fonction des questions électorales débattues au cours de la campagne (surtout la question de la pendaison de Louis Riel), - elle n'est pas même celle où la participation électorale a été la plus importante (Tableau VIII), - mais doit être interprétée à partir du contexte global des élections qui l'ont précédée. 